Appl. Phys. A 48, 155-160 (1989)

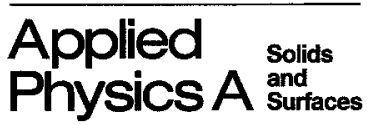

(C) Springer-Verlag 1989

\title{
Classification of Current Instabilities During Low-Temperature Breakdown in Germanium
}

\author{
J. Peinke ${ }^{1}$, J. Parisi ${ }^{1}$, B. Röhricht ${ }^{1}$, K. M. Mayer ${ }^{1}$, U. Rau ${ }^{1}$, W. Clauß ${ }^{1}$, R. P. Huebener ${ }^{1}$, \\ G. Jungwirt ${ }^{2}$, and W. Prettl ${ }^{2}$ \\ ${ }^{1}$ Physikalisches Institut, Lehrstuhl Experimentalphysik II, Universität, D-7400 Tübingen, \\ Fed. Rep. Germany \\ ${ }^{2}$ Institut für Physik III, Lehrstuhl Angewandte Physik, Universität, D-8400 Regensburg, \\ Fed. Rep. Germany
}

Received 19 May 1988/Accepted 21 September 1988

\begin{abstract}
We present experimental investigations on the spatio-temporal nonlinear current flow in the post-breakdown regime of p-germanium at liquid-helium temperatures. The basic nonlinear effects are characterized in terms of the underlying semiconductor physics, taking into account the influence of different experimental parameters.
\end{abstract}

PACS: $05.45 .+\mathrm{b}, 72.20 . \mathrm{Ht}, 72.70 .+\mathrm{m}$

It is well known that a large number of physical and nonphysical systems show spontaneous formation of spatial or temporal structures as a result of instability. Close to such instability points the dynamics of the system and its emerging structures are determined by a set of, in general, a few collective variables, often called order parameters. The underlying synergetic approach introduced by Haken [1] can explain the unexpected order and coherence arising on the macroscopic scale, regardless of the large number of competing physical forces interacting on the microscopic scale. Motivation for the intensive study of cooperative dynamics and pattern formation phenomena during the past few years derives from an increasing appreciation of the remarkable diversity of behavior encountered in nonlinear systems and of universal features shared by entire classes of similar nonlinear dynamic processes.

So far, it appears that the subject of such complex nonlinear behavior is dominated by theoretical investigations and computer studies, whereas experimental measurements on real physical systems represent the minority. Among the various objects which can be studied experimentally, solid-state turbulence in semiconductors appears particularly interesting [2]. Nonlinear current transport behavior during lowtemperature avalanche breakdown of extrinsic germanium comprises the self-sustained development of spatio-temporal dissipative structures in the formerly homogeneous semiconductor [3]. This kind of nonequilibrium phase transition between different conducting states results from the autocatalytic nature of impurity impact ionization generating mobile charge carriers [4]. The simple and direct experimental accessibility via advanced measurement techniques favors semiconductors as a nearly ideal study object for complex nonlinear dynamics compared to other physical systems. Further representing a convenient model reaction-diffusion system that exhibits distinct universal features, the present semiconductor system may acquire general significance for many synergetic systems in nature. Finally, in view of the rapidly growing application of semiconductor technologies, the understanding, control, and possible exploitation of sources of instability in these systems have considerable practical importance.

This paper gives a classification of our experimental investigations on the spatio-temporal nonlinear current flow in the post-breakdown regime of p-germanium at liquid-helium temperatures. Section 1 briefly outlines an example of a set of nonlinear current instabilities obtained from our semiconductor system. Section 2 reports the characterization of the basic nonlinearities in the light of the underlying physics and the relevant control parameters. Finally, Sect. 3 
touches upon the complex interplay between microscopic physics and macroscopic system behavior observed experimentally.

\section{Typical Instabilities}

Our experimental system consists of single-crystalline p-doped germanium, electrically driven into lowtemperature avalanche breakdown via impurity impact ionization. The typical sample geometry and the electronic measuring configuration are sketched in Fig. 1. Having dimensions of about $0.2 \times 2 \times 5 \mathrm{~mm}^{3}$ and an acceptor concentration of about $10^{14} \mathrm{~cm}^{-3}$, the extrinsic germanium crystal carries properly arranged ohmic aluminum contacts placed on one of the two largest surfaces. To provide the outer ohmic contacts with an electric field, a dc bias voltage $V_{0}$ was applied to the series combination of the sample and the load resistor $R_{\mathrm{L}}$. A dc magnetic field parallel or perpendicular to the broad sample surfaces could also be applied by a superconducting solenoid surrounding the semiconductor sample. The resulting electric current $I$ was found from the voltage drop at the load resistor. The voltage $V$ was measured along the sample. The inner probe contacts (of about $0.2 \mathrm{~mm}$ diameter) served as an independent monitor of the partial voltages $V_{i}(i=1,2,3)$ along the sample. Utilizing lowtemperature scanning electron microscopy [5], twodimensional images of spatial current patterns were obtained by scanning the specimen surface with an electron beam and by recording the beam-induced current change in the voltage-biased specimen as a function of the beam coordinate $(x, y)$. During the experiments, the semiconductor sample was kept at liquid-helium temperatures ( $4.2 \mathrm{~K}$ or below) and carefully protected against external electromagnetic irradiation (visible, far infrared).

The autocatalytic process of impurity impact ionization is reflected in a strongly nonlinear curvature of the measured current-voltage characteristic (sometimes with $S$-shaped negative differential resistance) in

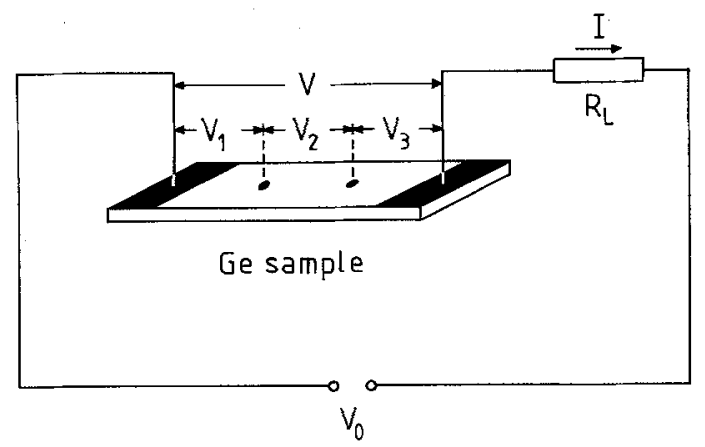

Fig. 1. Scheme of the typical experimental set-up. The shaded areas on the $\mathrm{Ge}$ sample indicate the evaporated ohmic $\mathrm{Al}$ contacts

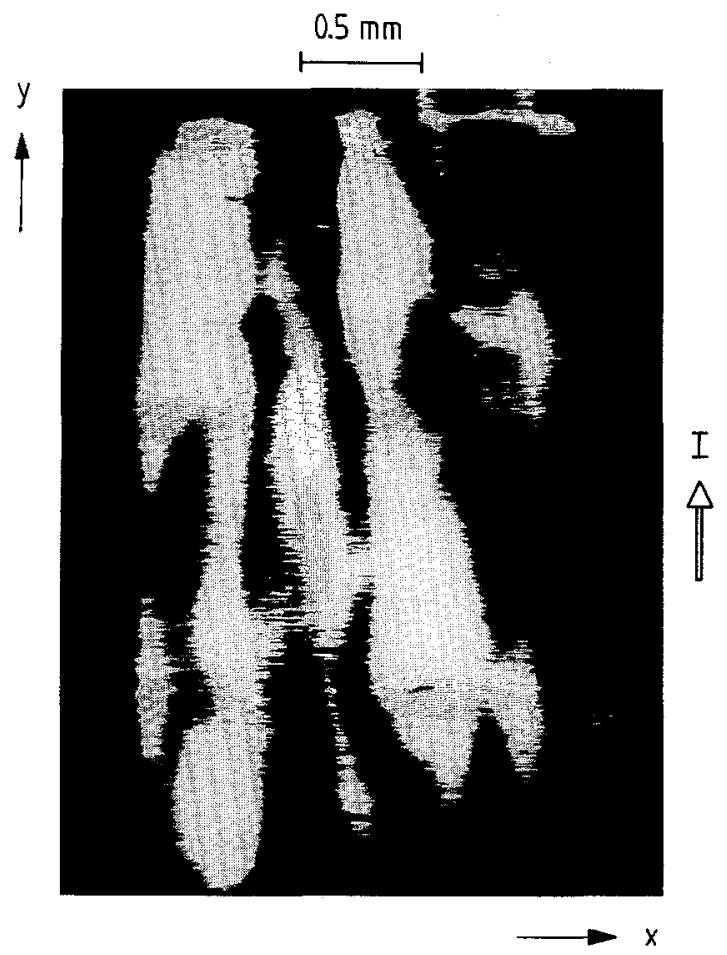

Fig. 2. Brightness-modulated image of the filamentary current flow in a homogeneously doped Ge sample during avalanche breakdown obtained by low-temperature scanning electron microscopy (load resistor $R_{\mathrm{L}}=1 \Omega$, bias voltage $V_{0}=2.00 \mathrm{~V}$, magnetic field $B=0 \mathrm{G}$, bath temperature $T=4.2 \mathrm{~K}$, no electromagnetic irradiation). The dark regions correspond to the filament channels extending along the $y$-direction

the immediate post-breakdown regime $[4,6]$. Due to the inherent multiplication of mobile charge carriers during avalanche breakdown, the resulting current flow drastically increases by several orders of magnitude (typically, from a few $n A$ in the pre-breakdown up to a few $\mathrm{mA}$ in the post-breakdown region). Simultaneously, spontaneous emergence of both spatial and temporal dissipative structures in the electric carrier transport takes place.

The complex spatial behavior of our semiconductor system can be globally visualized by means of lowtemperature scanning electron microscopy. Figure 2 shows a two-dimensional image of a typical current filament pattern developing in the nonlinear regime of the current-voltage characteristic. As reported elsewhere [7] in detail, the multifilamentary current flow becomes more and more homogeneous if the semiconductor system is driven further into its linear postbreakdown region at higher electric fields. Nucleation of additional filaments is often accompanied by abrupt changes between different stable filament configurations via noisy current instabilities.

The highly nonlinear current-voltage curve is further associated with the appearance of self-generated 

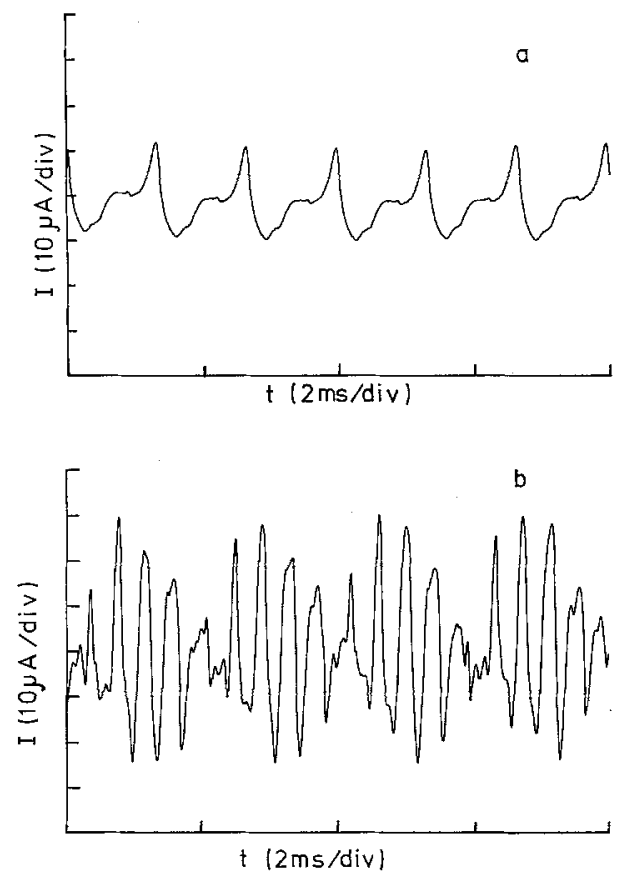

Fig. 3a, b. Temporal structure of spontaneous current oscillations superimposed upon the steady dc current in the postbreakdown regime of the Ge sample described in Fig. 1 (load resistor $R_{\mathrm{L}}=100 \Omega$, bias voltage $V_{0}=2.33 \mathrm{~V}$, transverse magnetic field $B$ varied, bath temperature $T=2.1 \mathrm{~K}$, no electromagnetic and no electron-beam irradiation): a periodic state $(B=5.3 \mathrm{G}) ; \mathbf{b}$ quasiperiodic state $(B=19.1 \mathrm{G})$

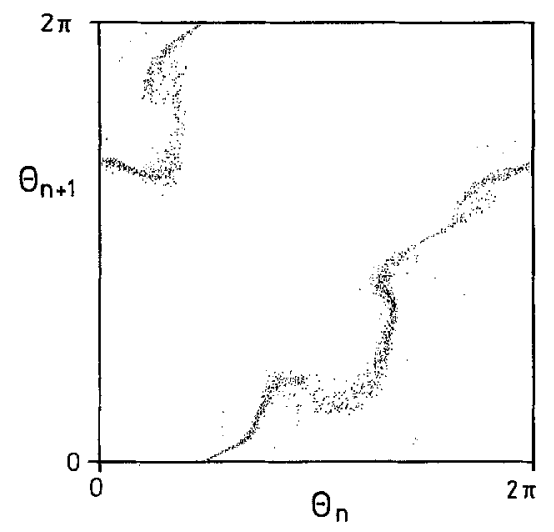

Fig. 4. Return map of a chaotic state (load resistor $R_{\mathrm{L}}=8.392 \mathrm{k} \Omega$, bias voltage $V_{0}=12.00 \mathrm{~V}$, transverse magnetic field $B=20.7 \mathrm{G}$, bath temperature $T=2.0 \mathrm{~K}$, no electromagnetic and no electronbeam irradiation) constructed from the projection of the strobed temporal current signal onto the angular variable. The method is outlined in the text

current and voltage oscillations. Both current $I$ and partial voltages $V_{i}(i=1,2,3)$ display - superimposed upon the dc signals of typically a few $\mathrm{mA}$ and some hundred $\mathrm{mV}$, respectively - temporal oscillations with a relative amplitude of about $10^{-3}$ in the frequency range $0.1-100 \mathrm{kHz}$. An example of spontaneous cur- rent oscillations is shown in Fig. 3. Parts (a) and (b) display a periodic and a quasiperiodic state of the underlying system attractor, respectively. By slightly varying certain control parameters (electric field, magnetic field, temperature, electromagnetic irradiation and/or electron-beam irradiation) the temporal behavior of the system variables $V_{1}, V_{2}, V_{3}$, and $I$ changes dramatically, exhibiting the typical universal scenarios of chaotic nonlinear systems [8-11]. An example of a chaotic state is shown in Fig. 4. As described elsewhere $[10,12]$, the reduced onedimensional return map $\theta_{n+1}$ vs. $\theta_{n}$ of the dynamic system behavior is generated by strobing the measured current signal $I(t)$ at internal drive phase and subsequently projecting the discrete current values $I_{n}$ $(n=1,2, \ldots$,$) onto the angular variable \theta_{n}$ via the relation

$\theta_{n}=\arctan \left(I_{n+1} / I_{n}\right)$.

Obviously, the map develops local minima and "wiggles", ceasing to be an invertible diffeomorphism and, thus, indicating chaotic behavior.

\section{System Characterization}

In the preceding part of this paper, we have demonstrated some typical nonlinear features obtained for the p-germanium system at low temperatures driven into a distinct breakdown regime. It should be pointed out that an inherent attribute of nonlinear systems is their high sensitivity to changes of the control parameters. Thus, good reproducibility of specific nonlinear effects could be observed only in measurements performed on one sample. Comparing results from different samples, only an overall agreement in the basic nonlinear behavior was found, i.e., electric breakdown, spontaneous oscillations, and current filamentation could generally be observed. In the following, these basic nonlinearities are characterized in terms of semiconductor physics by discussing the influence of different experimental parameters. In particular, we concentrate on the following aspects: material description, sample preparation, contact effects, length effects, and influences of the experimental set-up.

The samples investigated were prepared from single-crystalline $\mathrm{p}-\mathrm{Ge}$ slices. Two different series of similar material specifications were available, the crystallographic growth axes being oriented in the (100) and (111) direction, respectively. The specific resistivity at room temperature ranged from 10 to $20 \Omega \mathrm{cm}$, corresponding to an impurity concentration of $(2-3) \times 10^{14} \mathrm{~cm}^{-3}[13]$. The compensation ratios were definitely smaller than $5 \times 10^{-2}$. For a detailed specification of the acceptor material in question, we present in Fig. 5 a measurement of a typical photo- 


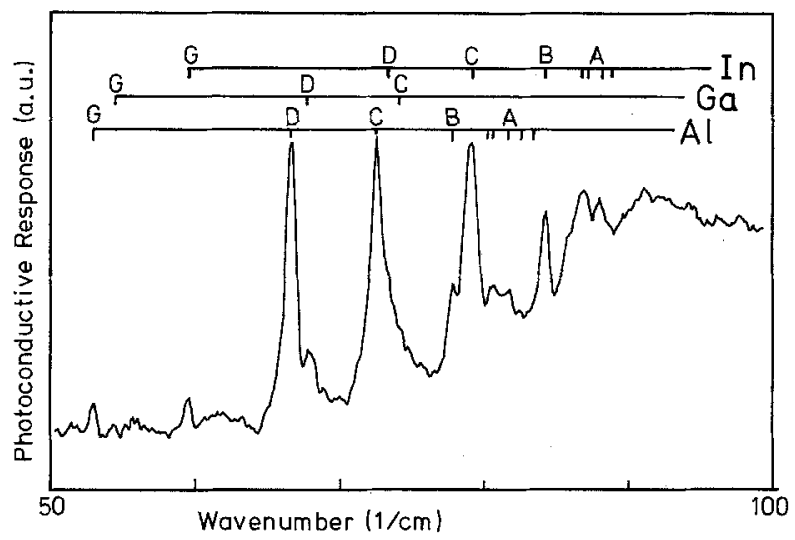

Fig. 5. Photoconductivity spectrum of one representative $\mathrm{Ge}$ sample obtained from the measured photocurrent signal as a function of the wavenumber of the exciting irradiation. Further details are given in the text and in [14]

conductivity spectrum. Comparing this spectrum with the spectrum of the energy levels of all possible acceptor materials [14] allows us to identify different acceptor materials present in our semiconductor samples. Here we obtained for one representative sample three different shallow acceptors, namely $\mathrm{Al}, \mathrm{In}$, and $\mathrm{Ga}$, each contributing at least $10 \%$ to the total impurity concentration (see Fig. 5). Taking into account that we have investigated a variety of different crystal samples and that the basic nonlinear features were observed in all cases, we conclude that the observed nonlinear effects are not linked to a particular acceptor concentration or crystal orientation. These findings are in agreement with similar chaos experiments performed on ultrapure $\mathrm{p}$-Ge by Teitsworth et al. [15] and Gwinn and Westervelt [16].

Next we turn to the sample preparation. Usually, the surfaces of the samples were successively polished and etched, in order to obtain an ideal surface structure. No significant influence on the basic nonlinearities due to different surface treatment could be observed, even if the etching procedure was totally omitted. Thus, we conclude that the basic nonlinear effects do not depend on the surface structure of the sample used.

As the next preparation step, ohmic contacts were put on the sample. For that we applied two different techniques, namely alloying and ion implantation. As an alloying material, we utilized $\mathrm{Al}$ (samples illustrated in Figs. 1-5), In, and a mixture of Ga and In. For obtaining ion-implanted contacts, an ion beam of ${ }^{11} \mathbf{B}$ with an acceleration energy of $60 \mathrm{keV}$ and a surface density of $5 \times 10^{14} \mathrm{~cm}^{-2}$ was used (sample in Fig. 6) [17]. Again, the application of different contact materials did not change the basic results. From this we conclude that the nonlinearities in our semiconductor system originate from a bulk effect and are not contact induced. This statement was further confirmed by experimental observations of localized oscillation centers in the bulk of the semiconductor sample $[3,7,10]$.

In addition to the influence of different contact materials, the influence of different contact geometries was systematically investigated. Contact shapes ranging from capacitor-like parallel planes to spike-like planar forms were arranged on the crystal surface. Here we found that different geometries of the facing contacts resulted in different shapes of the currentvoltage characteristic in the breakdown regime. Such phenomenon can be well understood if we assume the microscopic physics (current density versus electric field characteristic) to be independent of the particular contact geometry, but take into account macroscopic contact-form induced features $[6,18]$. Regardless of these changes in the current-voltage characteristics, the basic nonlinear effects could still be detected.

We have varied not only the geometry, but also the distance between the contacts. For relatively large distances in the range of some $\mathrm{mm}$, differently prepared contacts always led to more or less the same results. For distances in the range of some $100 \mu \mathrm{m}$ and smaller, only samples with ion-implanted contacts [17] showed the expected linear dependence of the breakdown voltage on the distance between the contacts [18]. We emphasize that we could observe breakdown and spontaneous oscillations even in samples with the shortest contact distance of $30 \mu \mathrm{m}$ (for example, Fig. 6). Furthermore, it should be noted that the typical frequency of the spontaneous oscillations did not change with decreasing contact distance (compare Fig. 6 with Fig. 3). Since the measured oscillation frequencies definitely showed no length dependence of any kind, the possible mechanism of travelling highfield domains can be excluded as an explanation of the spontaneous current oscillations. On the other hand, it

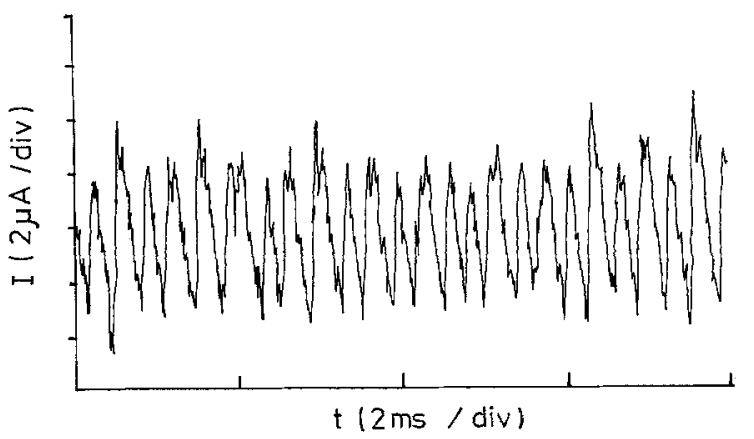

Fig. 6. Temporal structure of spontaneous current oscillations superimposed upon the steady dc current in the post-breakdown regime of a miniaturized $\mathrm{Ge}$ sample with $30 \mu \mathrm{m}$ contact distance (load resistor $R_{\mathrm{L}}=8.200 \mathrm{k} \Omega$, bias voltage $V_{0}=122.9 \mathrm{mV}$, transverse magnetic field $B=3.0 \mathrm{G}$, bath temperature $T=4.2 \mathrm{~K}$, no electromagnetic and no electron-beam irradiation) 
may be worth pointing out that the concept of filamentary current patterns appears to be limited to contact distances larger than $10 \mu \mathrm{m}[7,18]$.

So far, we have discussed effects resulting from differently prepared samples. Now we focus on the influence of external parameters. The spontaneous oscillations were generally found to be stable against changes of the experimental set-up, i.e., capacitances or inductances of the electronic circuit. Thus, circuitinduced oscillations could be excluded. On the other hand, the detailed temporal structure of the oscillations was found to be very sensitive to an external transverse magnetic field, whereas the existence of the oscillations was not affected. Although the sensitivity to a longitudinal magnetic field was much weaker, we generally observed a more systematic influence of this control parameter. For example, the oscillation frequencies always increased with increasing longitudinal magnetic field [19]. A similar frequency behavior was observed in the case where the power of an external (electromagnetic or electron-beam) irradiation applied to the sample was increased [20]. This control parameter also affected the shape of the currentvoltage characteristic. With increasing irradiation power the curve shifted towards lower electric fields and, additionally, increased less abruptly in the breakdown region $[6,20]$. Representing a further important control parameter, the temperature of the liquid-helium bath produced similar effects on the measured current-voltage characteristic. In particular, nonequilibrium phase transitions in the electronic transport behavior have recently been demonstrated as the temperature range was extended up to $10 \mathrm{~K}$ [21].

Finally, we turn to the physics of the breakdown mechanism underlying the nonlinear transport phenomena observed in our p-germanium system at low temperatures. As described elsewhere [22] in detail, we have determined the electric field dependence of the intrinsic system quantities (carrier density, mobility, and drift velocity) in the breakdown regime from conductivity and Hall-effect measurements. The breakdown characteristic was demonstrated to be drastically influenced by the variation of the carrier mobility which sensitively depends upon the density of the mobile charge carriers. Moreover, the quantitative evaluation of the transport properties throughout the breakdown region yielded more detailed information on the elementary scattering processes of the conduction carriers occurring in the present semiconductor system.

\section{Concluding Remarks}

There is much literature about the different mechanisms leading to instabilities in semiconductors (see [2]).
The best agreement with the present experimental results is obtained if we model our semiconductor system by a hole plasma generated via an autocatalytic avalanche breakdown mechanism. In order to explain negative differential current-voltage characteristics, Kastalsky [23] introduced a multilevel transport model for the charge carriers. The basic assumption is that the charge carriers can occupy the conduction band or can be bound to the ground and excited states of impurity centers. With an analogous rateequation model involving at least two impurity levels, Schöll $[4,24]$ explicitly derived analytical conditions for both filamentary and oscillatory instabilities. The central idea of "breathing" current filaments together with long-range coupling of spatially separated (localized) oscillation centers via energy exchange may roughly explain some of the oscillatory behavior of our samples. But, on the other hand, a detailed description of the wide variety of nonlinear effects, especially the complex chaotic behavior, cannot be expected on the basis of such physical models. As in turbulence, it seems to be impossible to describe the global system behavior starting from first principles. Furthermore, it is well known that complex nonlinear behavior can be modelled with an astonishingly high precision by universal nonlinear ad-hoc models.

The question remains of how the classification of a nonlinear dynamic system on the basis of the underlying physics can contribute to the understanding of the self-organized formation of complex dynamic structures. In the sense of Haken [1], we feel that all traditional disciplines in physics, which are concerned with the macroscopic behavior of multicomponent systems, require new ideas and concepts based on the synergetic approach, in order to cope with selforganizing systems.

Acknowledgements. We thank E. E. Haller, M. Duong-van, R. Friedrich, and R. Richter for helpful conversations and H. Leier, A. Forchel, and M. Pilkuhn for providing ion-implanted sample contacts. This work was supported financially in part by the Stiftung Volkswagenwerk (grants for J. Pe. and B. R.) and in part by the Deutsche Forschungsgemeinschaft (grants for K. M.M. and U.R.).

\section{References}

1. H. Haken: Advanced Synergetics, Springer Ser. Syn. Vol. 20 (Springer, Berlin, Heidelberg 1983)

2. R.P. Huebener, J. Peinke, J. Parisi: Appl. Phys. A 48, 107-110 (1989)

3. R.P. Huebener, K.M. Mayer, J. Parisi, J. Peinke, B. Röhricht: Nuclear Physics B (Proc. Suppl.) 2, 3 (1987)

J. Peinke, J. Parisi, B. Röhricht, K.M. Mayer, U. Rau, R.P. Huebener: Solid State Electron. 31, 817 (1988) 
4. E. Schöll: Nonequilibrium Phase Transitions in Semiconductors (Springer, Berlin, Heidelberg 1987)

E. Schöll, J. Parisi, B. Röhricht, J. Peinke, R.P. Huebener: Phys. Lett. 119 A, 419 (1987)

J. Parisi, J. Peinke, B. Röhricht, U. Rau, M. Klein, O.E. Rössler: Z. Naturforsch. 42a, 655 (1987)

5. R.P. Huebener: Rep. Prog. Phys. 47, 175 (1984)

6. J. Peinke, D.B. Schmid, B. Röhricht, J. Parisi: Z. Phys. B 66, 65 (1987)

7. K.M. Mayer, R. Gross, J. Parisi, J. Peinke, R.P. Huebener: Solid State Commun. 63, 55 (1987)

K.M. Mayer, J. Peinke, B. Röhricht, J. Parisi, R.P. Huebener: Phys. Scr. T 19, 505 (1987)

8. J. Peinke, A. Mühlbach, R.P. Huebener, J. Parisi: Phys. Lett. 108 A, 407 (1985)

9. J. Peinke, B. Röhricht, A. Mühlbach, J. Parisi, Ch. Nöldeke, R.P. Huebener, O.E. Rössler: Z. Naturforsch. 40a, 562 (1985)

10. J. Peinke, J. Parisi, B. Röhricht, B. Wessely, K.M. Mayer: Z. Naturforsch. 42a, 841 (1987)

11. U. Rau, J. Peinke, J. Parisi, R.P. Huebener, E. Schöll: Phys. Lett. 124 A, 335 (1987)

12. J. Peinke, J. Parisi, R.P. Huebener, M. Duong-van, P. Keller: Phys. Rev. Lett. (submitted)

13. S.M. Sze: Physics of Semiconductor Devices (Wiley Eastern, New Delhi 1979)
14. R.L. Jones, P. Fisher: J. Phys. Chem. Solids 26, 1125 (1965) E.E. Haller, W.L. Hansen: Solid State Commun. 15, 687 (1974)

L.S. Darken: J. Appl. Phys. 53, 3754 (1982)

15. S.W. Teitsworth, R.M. Westervelt, E.E. Haller: Phys. Rev. Lett. 51, 825 (1983)

S.W. Teitsworth, R.M. Westervelt: Phys. Rev. Lett. 56, 516 (1986)

16. E.G. Gwinn, R.M. Westervelt: Phys. Rev. Lett. 57, 1060 (1986)

E.G. Gwinn, R.M. Westervelt: Phys. Rev. Lett. 59, 157 (1987)

17. Boron-ion-implanted contacts were fabricated at the Microstructure Laboratory of the University of Stuttgart

18. W. ClauB: Unpublished

19. U. Rau, J. Peinke, J. Parisi, R.P. Huebener: Z. Phys. B 71, $305(1988)$

20. K.M. Mayer, J. Parisi, J. Peinke, R.P. Huebener: Physica $32 \mathrm{D}, 306$ (1988)

21. B. Röhricht, R.P. Huebener, J. Parisi, M. Weise: Phys. Rev. Lett. (submitted)

22. J. Parisi, U. Rau, J. Peinke, K.M. Mayer: Z. Phys. B 72, 225 (1988)

23. A.A. Kastalsky: Phys. Stat. Solidi (a) 15, 599 (1973)

24. E. Schöll: Physica 134 B, 271 (1985)

E. Schöll: Phys. Rev. B 34, 1395 (1986) 